\title{
Correction: Unraveling overlapping deletions by agglomerative clustering
}

Roland Wittler

From The Eleventh Asia Pacific Bioinformatics Conference (APBC 2013)

Vancouver, Canada. 21-24 January 2013

After the publication of my article [1], I noticed that Figure 2 shows the wrong graphic and Figure 7 was incorrect. The correct figures can be found here.

Published: 15 March 2013

\section{Reference}

1. Wittler R: Unraveling overlapping deletions by agglomerative clustering. BMC Genomics 2013, 14(Suppl 1):S12.

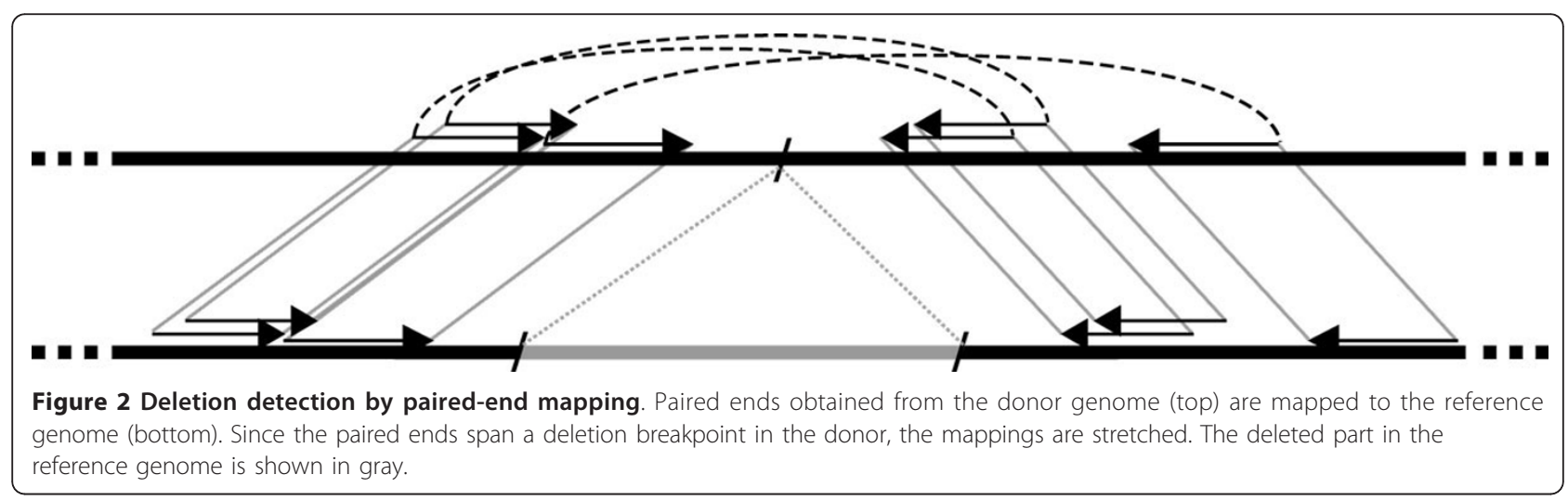

Correspondence: roland@CeBiTec.Uni-Bielefeld.DE

Genome Informatics, Faculty of Technology and Institute for Bioinformatics,

Center for Biotechnology (CeBiTec), Bielefeld University, 33594 Bielefeld, 


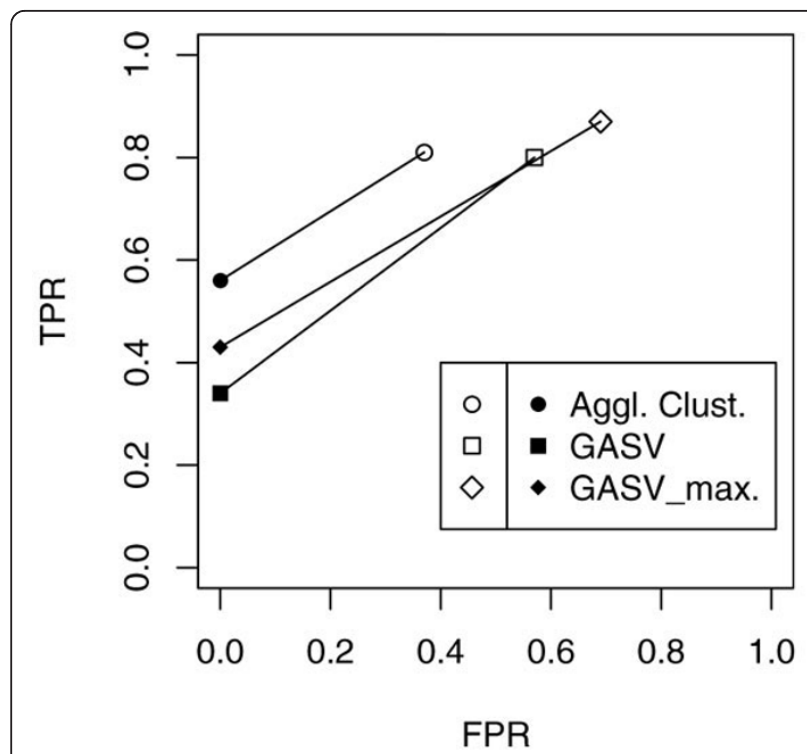

(a) Coverage 20, scenario A

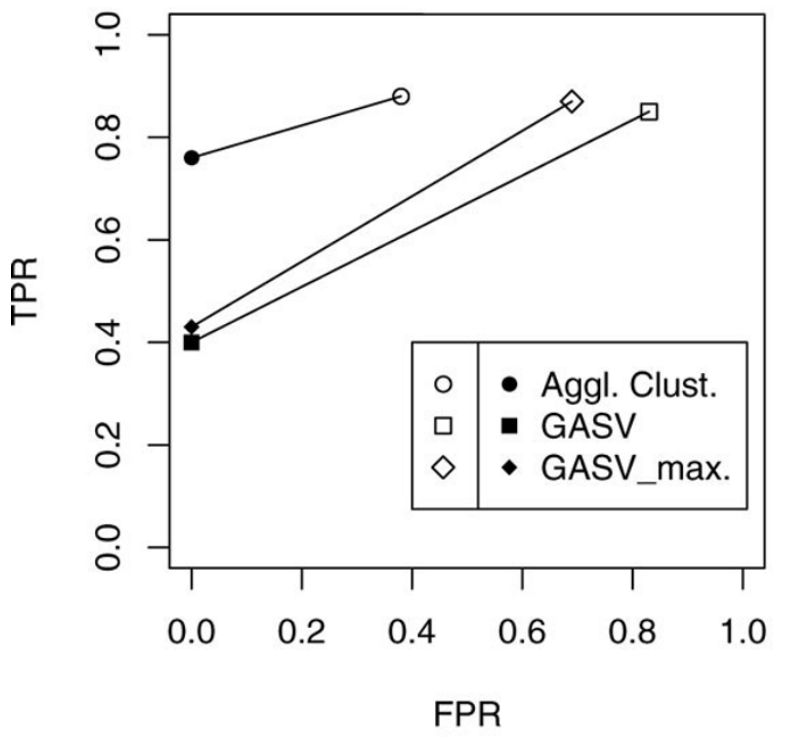

(c) Coverage 60, scenario A

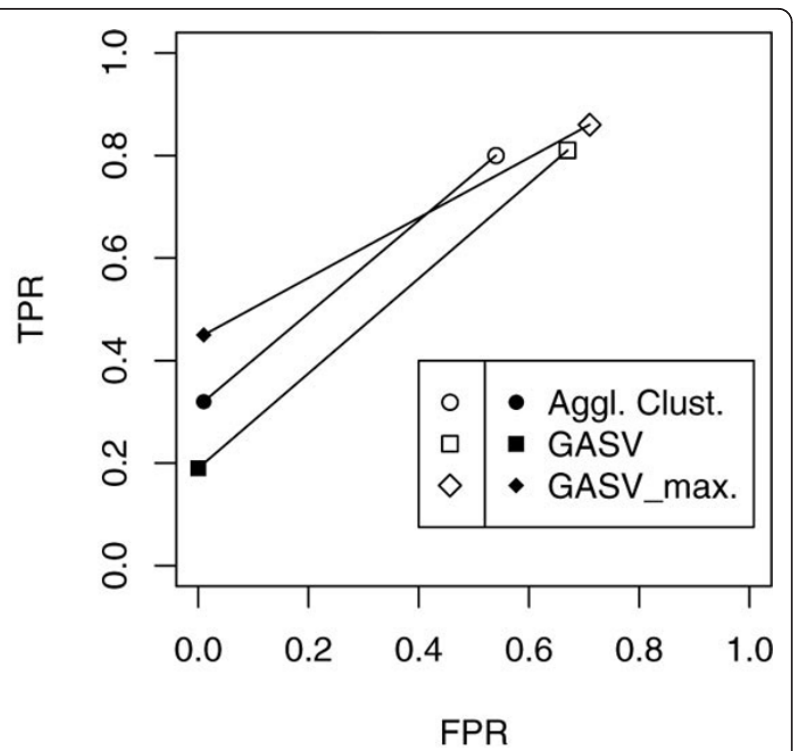

(b) Coverage 20, scenario B

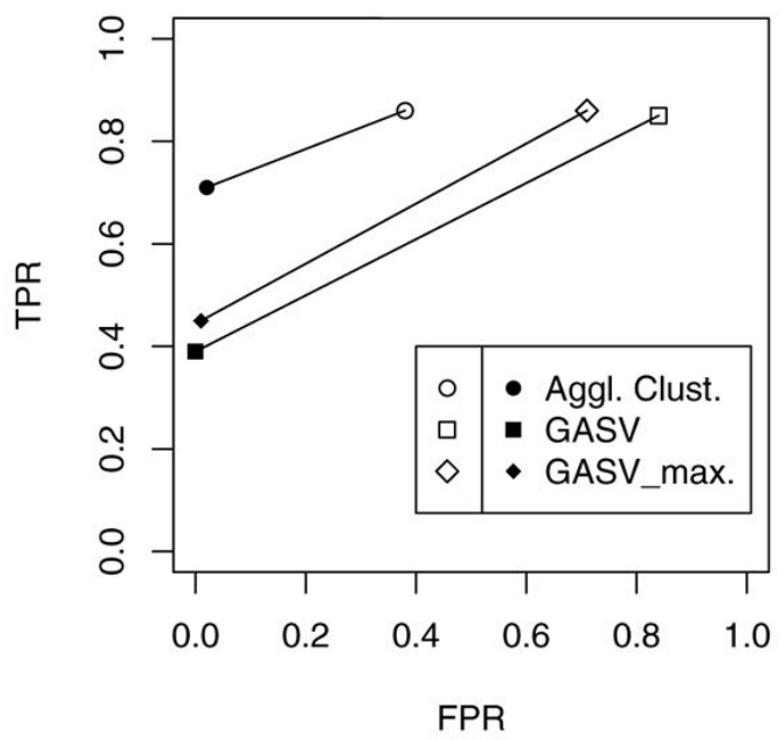

(d) Coverage 60, scenario B

Figure 7 Accuracy of different methods in detecting single or overlapping deletions. Accuracy of different methods in detecting single or overlapping deletions. For different coverages (20x and 60x), and two different simulated scenarios (see [[1], Figure 6]), the accuracy in

distinguishing single from overlapping deletions has been measured. The empty symbols represent the true and false positive rate for detecting single deletions (TPR1, FPR1). The solid symbols represent the true and false positive rate for detecting pairs of overlapping deletions (TPR2, FPR2). 